\title{
Exposure to Pro-eating Disorder Websites and Attitudes Towards Treatment
}

\author{
Benjamin Esser, Jennifer Lyke \\ The Richard Stockton College of New Jersey, Galloway, New Jersey, USA
}

\begin{abstract}
This study investigated whether variables associated with greater levels of involvement with pro-eating disorder Websites were related to more positive attitudes towards eating disorders and more negative attitudes towards their treatment. Participants were 149 individuals who identified themselves on pro-eating disorder Websites' "buddies" pages who completed a self-report questionnaire distributed via the Internet. The data was analyzed to determine whether the independent variables significantly predicted participants’ BMI (Body Mass Index) and/or their responses to four attitude questions. The amount of time spent on pro-eating disorder Websites and whether or not participants were members of these Websites significantly predicted participants' attitudes on three out of four questions related to eating disordered behavior and treatment of eating disorders. The results imply that involvement with pro-eating disorder Websites is significantly related to more positive attitudes towards eating disordered behavior and more negative attitudes towards treatment.
\end{abstract}

Keywords: pro-eating disorder, Websites, attitudes, eating behavior, treatment

\section{Introduction}

Through its ability to connect people who are geographically separate, the Internet has potential to help people access information, support and services for a variety of mental health issues. However, the same ability also potentially connects users with less positive effects. For example, the Internet can encourage suicidal behavior (Alao, Soderberg, Pohl, \& Alao, 2006), compulsive gambling (King, 1999) and sex addiction (Griffiths, 2001). Another example is an online movement that supports symptoms of eating disorders (Tierney, 2006). In the past several years, various pro-eating disorder Websites have emerged on the Internet that provide support or encouragement to visitors seeking to become thinner. These Websites take many different perspectives on symptoms of eating disorders, varying from active anti-recovery encouragement to simple support.

A recent study by Norris, Boydell, Pinhas, and Katzman (2006) attempted to analyze pro-eating disorder Websites to quantify their defining features and find commonalities between them. Many of the Websites have disclaimers about their content, warning that it may be offensive. This may be a response to webmasters who saw them as a liability and deleted them when the Websites first began to arise, or a response to hostile site visitors who have frequently attacked the communities (Giles, 2006).

Many pro-eating disorder Websites contain "buddies” pages where individuals post contact information, usually an e-mail address, so members can find like-minded people with whom they can fast, diet or just talk.

Benjamin Esser, School of Social and Behavioral Sciences, The Richard Stockton College of New Jersey.

Jennifer Lyke, Ph.D., School of Social and Behavioral Sciences, The Richard Stockton College of New Jersey. 
“Thinspiration”, another common feature of pro-eating disorder Websites, features pictures of very thin women, usually models, as inspiration to site visitors. "Tips and tricks” pages are another common feature of such Websites, listing ways to lose weight fast and fight off hunger. Two good examples of the pro-eating disorder movement online that show these different perspectives are http://www.pro-ana-nation.com and http://www. proanamia.com (Norris et al., 2006).

Although there has been some research done into the nature of these Websites, the effect of these Websites on individuals who view them is largely unknown. Since communities of eating disordered individuals did not previously exist outside clinical settings, the existence of these Websites may create a peer group that has an effect on users' attitudes towards eating disorder symptoms and their treatment. Research done outside the context of the pro-eating disorder movement by Lieberman, Gauvin, Bukowski, and White (2001) suggested that females are significantly affected by pressure from their peers in ways that negatively affect their body esteem and eating behaviors. Added to this pressure, the anonymous nature of online relationships may encourage disinhibition of negative impulses (Suler, 2004).

In addition, patients have suggested that pressure from partners, acquaintances and the "thin ideal" presented by the media make recovery difficult (Murray, Touyz, \& Beumont, 1995). Certainly, these sites may have a negative effect on treatment or the seeking of treatment given the combination of the peer group in this online community, which is likely to be predominantly female, and the presentation of "thinspiration" and other graphic methods of glorifying thinness (Tierney, 2006).

Another study of pro-eating disorder Websites by Bardone-Cone and Cass (2006) found that women who viewed these Websites decreased in self-esteem, self-efficacy and perceived attractiveness. They also increased in the perception of being overweight. However, a study investigating the linguistic differences between pro-anorexics and recovering anorexics found that pro-anorexics displayed more positive emotions, less anxiety, a lower degree of cognitive reflection, and lower levels of self-directed attention than did recovering anorexics (Lyons, Mehl, \& Pennebaker, 2006), indicating that the support provided by pro-eating disorder Websites may appeal to women with eating disorder symptoms, because they provide a relief from the painful or difficult aspects of recovery.

A fundamental question is whether these Websites have significant effects on the people who visit them. In particular, it is important to determine whether exposure to these Websites is associated with more positive attitudes towards eating disorders and negative attitudes towards treatment. The present study investigated whether variables associated with levels of involvement with pro-eating disorder Websites are related to attitudes towards eating disorders and their treatment.

\section{Method}

\section{Participants}

Participants were 149 individuals who identified themselves on pro-eating disorder Websites' "buddies" pages. They were contacted through publicly posted e-mail addresses. Approximately 4,000 emails were sent out to possible participants. One hundred forty-nine respondents who completely and properly completed the questionnaire were included in this study.

\section{Instruments}

Participants completed a self-report questionnaire which was distributed via the Internet. The questionnaire asked for the participant's age, race/ethnicity, gender, height, weight, information about their 
involvement with pro-eating disorder Websites and attitudes towards anorexia and bulimia. Specific questions related to the amount of involvement with pro-eating disorder Websites included whether they were currently a member of a pro-eating disorder Website, how many months they had been visiting pro-eating disorder Websites, the number of friends the participant had from the Websites and how much time per day they spent on the Websites. Specific questions related to attitudes towards eating disorders involved participants answering in a seven-point Likert scale the degree to which they agreed with the statements: "Anorexia and bulimia are medical and mental disorders"; "Individuals have the right to be anorexic or bulimic if they want"; "The mental health field should be treating people with anorexia and bulimia whether or not they want treatment”; and "Anorexia and bulimia can be healthy lifestyles if done correctly".

\section{Procedure}

The data was analyzed using several regression analyses to determine whether a significant relationship between the independent variables of membership, number of months as a visitor, number of friends and amount of time per day spent on the Websites significantly predicted participants' BMI (Body Mass Index) and/or their responses to the four attitude questions.

\section{Results}

\section{Descriptive Statistics}

The sample was 69.6\% Caucasian, 4.4\% Black, 3.7\% Hispanic, 2.2\% Asian, 1.5\% Native American and 4.4\% Other/Mixed, with $14.1 \%$ who did not report race/ethnicity. The sample was $98.5 \%$ female with $1.5 \%$ who did not report gender. The reported age of participants ranged from 12 to 41 with a mode of 18 years (14.1\%) and a mean of 19.89 years.

The majority (83.2\%) of the sample were members of a pro-eating disorder Website with the number of months visiting pro-eating disorder Websites ranging from one to $84(M=23.84, S D=20.14)$. Number of friends from the Websites ranged from zero to $60(M=4.79, S D=8.76)$. Average amount of time per day spent on the Websites ranged from zero to nine $(M=1.66, S D=1.56)$.

Independent samples $t$-test comparing members to non-members indicated that members have significantly more friends through pro-eating disorder Websites than non-members $\left(t_{(132)}=2.29, p<0.05\right)$ and spend significantly more time on these Websites than non-members do $\left(t_{(124)}=2.01, p<0.05\right)$. Bivariate correlations also indicated that number of months as a visitor was significantly positively correlated with number of friends through pro-eating disorder Websites $\left(r_{(125)}=0.213, p<0.05\right)$ and time spent per day in hours was significantly positively correlated with number of friends through pro-eating disorder Websites $\left(r_{(125)}=0.427, p<0.001\right)$.

\section{Data Screening}

Number of months as a visitor, number of friends from the Websites and time per day spent on the Websites were all missing significant amounts of data (5.4\%, 10.07\% and 15.43\%, respectively). Missing values were therefore replaced with the series mean for these variables. There were also four outliers with BMIs greater than 33 who were deleted from the data set. There were five outliers who reported visiting pro-eating disorder Websites for more than 72 months who were deleted from the data set. There were also 15 outliers who reported more than 13 friends from pro-eating disorder Websites. These cases were recoded into the tail of the distribution. There were seven outliers who reported more than seven hours per day visiting pro-eating disorder Websites. These cases were deleted from the data set. There were no multivariate outliers. 
BMI, number of months a visitor, number of friends from pro-eating disorder Websites and time per day spent on pro-eating disorder Websites were all positively skewed and were therefore transformed to improve normality. BMI and time per day were transformed using a square root function. Number of months and number of friends were transformed using a logarithm function.

\section{Multiple Regression Results}

Multiple regression analyses were performed to determine whether the independent variables (member of an eating disorder Website, number of months a visitor, number of friends and time per day) significantly predicted BMI and/or responses to the four attitude questions.

BMI. Regression results indicated that the predictors did not significantly predict BMI $\left(R^{2}=0.048\right.$, $R_{\text {adjusted }}^{2}=0.017, F_{(4,126)}=1.573, p>0.05$ ) (see Table 1 ).

Table 1

Summary of Multiple Regression Analysis for Variables Predicting BMI $(N=131)$

\begin{tabular}{lrrrrrr}
\hline Predictor & $B$ & \multicolumn{1}{l}{$\beta$} & \multicolumn{1}{l}{$t$} & \multicolumn{1}{l}{ Bivariate $r$} & Partial $r$ \\
\hline Member & 0.033 & 0.030 & 0.324 & 0.746 & -0.028 & 0.029 \\
Months & -0.041 & -0.036 & -0.397 & 0.692 & 0.034 & -0.035 \\
Friends & 0.277 & 0.252 & 2.452 & 0.016 & 0.201 & 0.213 \\
Time & -0.135 & -0.084 & -0.883 & 0.379 & 0.011 & -0.078 \\
\hline
\end{tabular}

Notes. $B$ : an unstandardized $B$ weight for each predictor in the multiple regression; $\beta$ : the standardized beta weight for each predictor; $t$ : the statistic testing whether the variable is a significant predictor in the equation; $p$ : the alpha level related to $t$, i.e., the indicator of whether the $t$-test is significant or not; $r$ : the statistic indicating a correlation.

Attitude question 1. The first attitude question was "Anorexia and bulimia are medical and mental disorders”. A multiple regression indicated the predictors significantly predicted participants' responses to this question $\left(R^{2}=0.113, R^{2}\right.$ adjusted $\left.=0.085, F_{(4,125)}=3.992, p<0.01\right)$. In this case, the only significant predictor was the average amount of time participants spent visiting pro-eating disorder Websites with amount of time spent on the Websites being negatively related to endorsement of the statement (see Table 2).

Table 2

Summary of Multiple Regression Analysis for Variables Predicting Attitudes Towards "Anorexia and Bulimia Are Medical and Mental Disorders" $(N=130)$

\begin{tabular}{llrrrrr}
\hline Predictor & $B$ & \multicolumn{1}{l}{$\beta$} & \multicolumn{1}{l}{$p$} & Bivariate $r$ & Partial $r$ \\
\hline Member & 0.177 & 0.033 & 0.372 & 0.710 & 0.054 & 0.033 \\
Months & 0.292 & 0.053 & 0.599 & 0.550 & 0.095 & 0.054 \\
Friends & 0.652 & 0.122 & 1.228 & 0.222 & -0.010 & 0.109 \\
Time & -2.711 & -0.350 & -3.773 & 0.000 & -0.308 & -0.320 \\
\hline
\end{tabular}

Notes. $B$ : an unstandardized $B$ weight for each predictor in the multiple regression; $\beta$ : the standardized beta weight for each predictor; $t$ : the statistic testing whether the variable is a significant predictor in the equation; $p$ : the alpha level related to $t$, i.e., the indicator of whether the $t$-test is significant or not; $r$ : the statistic indicating a correlation.

Attitude question 2. The second attitude question was, "Individuals have the right to be anorexic or bulimic if they want". The multiple regression results indicated that the predictors also significantly predicted participants' responses to this question $\left(R^{2}=0.093, R_{\text {adjusted }}^{2}=0.063, F_{(4,124)}=3.164, p<0.05\right)$. In this case, the only significant predictor was whether or not participants were members of a pro-eating disorder Website, with non-members being significantly more likely to endorse the statement than members (see Table 3). 
Table 3

Summary of Multiple Regression Analysis for Variables Predicting Attitudes Towards "Individuals Have the Right to Be Anorexic or Bulimic if They Want" $(N=129)$

\begin{tabular}{lrrrrrr}
\hline Predictor & $B$ & $\beta$ & $t$ & $p$ & Bivariate $r$ & Partial $r$ \\
\hline Member & -1.072 & -0.265 & -2.948 & 0.004 & -0.277 & -0.256 \\
Months & 0.365 & 0.087 & 0.967 & 0.336 & 0.076 & 0.086 \\
Friends & -0.006 & -0.002 & -0.016 & 0.987 & 0.133 & -0.001 \\
Time & 0.566 & 0.096 & 1.025 & 0.307 & 0.134 & 0.092 \\
\hline
\end{tabular}

Notes. $B$ : an unstandardized $B$ weight for each predictor in the multiple regression; $\beta$ : the standardized beta weight for each predictor; $t$ : the statistic testing whether the variable is a significant predictor in the equation; $p$ : the alpha level related to $t$, i.e., the indicator of whether the $t$-test is significant or not; $r$ : the statistic indicating a correlation.

Attitude question 3. The third attitude question was "The mental health field should be treating people with anorexia and bulimia whether or not they want treatment”. The multiple regression results indicated that the predictors did not significantly predict participants' responses to this question $\left(R^{2}=0.025, R_{\text {adjusted }}^{2}=-0.007\right.$, $F_{(4,123)}=0.776, p>0.05$ ) (see Table 4).

Table 4

Summary of Multiple Regression Analysis for Variables Predicting Attitudes Towards "The Mental Health Field Should Be Treating People With Anorexia and Bulimia Whether or Not They Want Treatment" $(N=128)$

\begin{tabular}{lrrrrrr}
\hline Predictors & $B$ & $\beta$ & \multicolumn{1}{l}{ B } & $p$ & Bivariate $r$ & Partial $r$ \\
\hline Member & 0.345 & 0.440 & 0.784 & 0.435 & 0.106 & 0.070 \\
Months & 0.144 & 0.464 & 0.311 & 0.756 & -0.001 & 0.028 \\
Friends & -0.513 & -0.110 & -1.043 & 0.299 & -0.132 & -0.094 \\
Time & -0.187 & -0.028 & -0.281 & 0.779 & -0.085 & -0.025 \\
\hline
\end{tabular}

Notes. $B$ : an unstandardized $B$ weight for each predictor in the multiple regression; $\beta$ : the standardized beta weight for each predictor; $t$ : the statistic testing whether the variable is a significant predictor in the equation; $p$ : the alpha level related to $t$, i.e., the indicator of whether the $t$-test is significant or not; $r$ : the statistic indicating a correlation.

Attitude question 4. The fourth attitude question was "Anorexia and bulimia can be healthy lifestyles, if done correctly". In this case, multiple regression results indicated that the combination of predictors significantly predicted participants' responses to this question $\left(R^{2}=0.131, R_{\text {adjusted }}^{2}=0.103, F_{(4,125)}=4.722, p\right.$ $<0.01$ ). Both whether the participant was a member of a pro-eating disorder Website and the average amount of time per day spent on these Websites were significantly related to endorsement of this question (see Table 5). Non-members were less likely to endorse this statement than members and the longer participants spent on these Websites, the more likely they were to endorse this statement.

Table 5

Summary of Multiple Regression Analysis for Variables Predicting Attitudes Towards "Anorexia and Bulimia Can Be Healthy Lifestyles if Done Correctly" $(N=130)$

\begin{tabular}{lllllll}
\hline Predictor & $B$ & $\beta$ & $t$ & $p$ & Bivariate $r$ & Partial $r$ \\
\hline Member & -1.742 & -0.290 & -3.318 & 0.001 & -0.292 & -0.285 \\
Months & 0.932 & 0.539 & 1.727 & 0.087 & 0.118 & 0.153 \\
Friends & -0.457 & -0.077 & -0.778 & 0.438 & 0.119 & -0.069 \\
Time & 1.638 & 0.189 & 2.061 & 0.041 & 0.200 & 0.181 \\
\hline
\end{tabular}

Notes. $B$ : an unstandardized $B$ weight for each predictor in the multiple regression; $\beta$ : the standardized beta weight for each predictor; $t$ : the statistic testing whether the variable is a significant predictor in the equation; $p$ : the alpha level related to $t$, i.e., the indicator of whether the $t$-test is significant or not; $r$ : the statistic indicating a correlation. 


\section{Discussion and Conclusions}

The results of this study imply that amount of involvement with pro-eating disorder Websites may significantly affect some attitudes towards eating disorders and their treatment. The beliefs that anorexia and bulimia are not mental disorders and can be done correctly are certainly problematic given the ample evidence for harmful consequences of these behaviors.

These findings have serious implications, even though the results are only correlational. Certainly, it may be that one direction of influence in this association is that young women with a greater propensity towards eating disordered behavior tend to spend more time on these sites. However, it is also likely that the amount of time spent on these sites has some influence on the way users think about these behaviors. The positive association found between the amount of time individuals spend on pro-eating disorder Websites and the number of friends they have from the Websites suggests that people who spend more time on these Websites may have more friends who share negative attitudes towards treatment of eating disorders. Having more pro-eating disorder friends could make recovery difficult and negatively impact the self-esteem and self-efficacy of treatment-oriented individuals.

Another interesting result of this study is that time spent on pro-eating disorder Websites did not significantly predict BMI. Variability in BMI may have obscured this result. Websites used as sources for participant e-mails included sites that support both anorexia and bulimia, but future research should target only pro-anorexia sites to confirm this finding.

There are several limitations to this study which are important to keep in mind when interpreting the results. First, the very small response rate is a serious liability to the validity of these results, since the sample may not adequately represent the population of interest. However, it may be that e-mail addresses had changed or that e-mails from an unfamiliar source were routed to junk mail folders, so it is difficult to gauge the actual response rate.

Another limitation to this study is that important variables such as time spent on the Websites and those used to calculate BMI were self-reported by participants. Also, there is no established validity to the survey instrument and variability in responses may be due to the need for more specificity in the survey questions, especially with respect to the severity of the symptoms referred to in the questions.

In spite of these limitations, the results of this study indicate that pro-eating disorder Websites may influence visitors in complex ways that should be examined in future research. For example, online communication may be especially appealing to eating disordered people who are socially anxious. Future research should investigate personality characteristics and co-morbid conditions that may interact with exposure to these sites to affect individuals' attitudes towards treatment.

The influence of the media on eating disordered behavior is well established. As a relatively new form of media, the Internet has unique characteristics that may aggravate the negative impact of such media messages, such as anonymity and lack of editorial oversight. However, the Internet may also offer unique advantages to therapeutic work with "shame-based" problems like eating disorders (Skarderud, 2003). What is clear is that there is an inherent risk to vulnerable individuals who are exposed to a community that supports disordered behavior. Future research should be targeted at more explicitly identifying ways these Websites impact their visitors and the long-term consequences for treatment.

\section{References}

Alao, A. O., Soderberg, M., Pohl, E. L., \& Alao, A. L. (2006). Cybersuicide: Review of the role of the internet on suicide. CyberPsychology and Behavior, 9(4), 489-495. 
Bardone-Cone, A. M., \& Cass, K. M. (2006). Investigating the impact of pro-anorexia Websites: A pilot study. European Eating Disorders Review, 14(4), 256-262.

Giles, D. (2006). Constructing identities in cyberspace: The case of eating disorders. British Journal of Social Psychology, $45,463-477$.

Griffiths, M. (2001). Sex on the Internet: Observations and implications for Internet sex addiction. The Journal of Sex Research, $38(4), 333-342$.

King, S. A. (1999). Compulsive Internet gambling: A new form of an old clinical pathology. CyberPsychology and Behavior, 2(5), 441-456.

Lieberman, M., Gauvin, L., Bukowski, W. M., \& White, D. R. (2001). Interpersonal influence and disordered eating behaviors in adolescent girls: The role of peer modeling, social reinforcement and body-related teasing. Eating Behaviors, 2(3), 215-236.

Lyons, E. J., Mehl, M. R., \& Pennebaker, J. W. (2006). Pro-anorexics and recovering anorexics differ in their linguistic Internet self-presentation. Journal of Psychosomatic Research, 60(3), 253-256.

Murray, S. H., Touyz, S. W., \& Beumont, P. J. V. (1995). The influence of personal relationships on women. Eating Disorders: The Journal of Treatment and Prevention, 3(30), 243-252.

Norris, M. L., Boydell, K. M., Pinhas, L., \& Katzman, D. K. (2006). Ana and the Internet: A review of pro-Anorexia Websites. International Journal of Eating Disorders, 39(6), 443-447.

Skarderud, F. (2003). Sh@me in cyberspace. Relationships without faces: The E-media and eating disorders. European Eating Disorders Review, 11, 155-169.

Suler, J. (2004). The online disinhibition effect. CyberPsychology and Behavior, 7(3), 321-326.

Tierney, S. (2006). The dangers and draw of online communication: Pro-anorexia Websites and their implications for users, practitioners, and researchers. Eating Disorders: The Journal of Treatment and Prevention, 14(3), 181-190. 